\title{
ADDITIVE ENERGY AND IRREGULARITIES OF DISTRIBUTION
}

\author{
Christoph Aistleitner — Gerhard LARCher
}

\begin{abstract}
We consider strictly increasing sequences $\left(a_{n}\right)_{n>1}$ of integers and sequences of fractional parts $\left(\left\{a_{n} \alpha\right\}\right)_{n>1}$ where $\alpha \in \mathbb{R}$. We show that a small additive energy of $\left(a_{n}\right)_{n>1}$ implies that for almost all $\alpha$ the sequence $\left(\left\{a_{n} \alpha\right\}\right)_{n>1}$ has large discrepancy. We prove a general result, provide various examples, and show that the converse assertion is not necessarily true.
\end{abstract}

Communicated by Michael Drmota

\section{Introduction and statement of results}

Let $\left(a_{n}\right)_{n \geq 1}$ be a strictly increasing sequence of positive integers. We are interested in distribution properties of the sequence $\left(\left\{a_{n} \alpha\right\}\right)_{n>1}$, where $\alpha$ is a given real and $\{x\}$ denotes the fractional part of $x$. In particular we are interested in the behaviour of the star-discrepancy $D_{N}^{*}$ of these sequences from a metrical point of view. The star-discrepancy $D_{N}^{*}$ of the first $N$ elements of a sequence $\left(x_{n}\right)_{n \geq 1}$ in $[0,1)$ is defined by

$$
D_{N}^{*}\left(x_{1}, \ldots, x_{N}\right):=\sup _{0<\beta \leq 1}\left|\frac{A_{N}(\beta)}{N}-\beta\right|,
$$

where $A_{N}(\beta):=\#\left\{1 \leq n \leq N \mid x_{n} \in[0, \beta)\right\}$. The sequence $\left(x_{n}\right)_{n \geq 1}$ is uniformly distributed in $[0,1)$ if and only if $\lim _{N \rightarrow \infty} D_{N}^{*}=0$.

There exists a vast literature on the discrepancy of sequences of the form $\left(\left\{a_{n} \alpha\right\}\right)_{n \geq 1}$. The most basic and classical example for such a class of sequences

2010 Mathematics Subject Classification: 11J83, 11K38, 11B30, 11B13, 11L03.

Keywords: discrepancy, additive energy, metric number theory, additive combinatorics.

The first author is supported by the Austrian Science Fund (FWF) project I1751-N26. Both authors are supported by the FWF project F5507-N26, which is part of the Special Research Program "Quasi-Monte Carlo Methods: Theory and Applications". 
are the Kronecker sequences $(\{n \alpha\})_{n \geq 1}$. It is well known that for the discrepancy of the Kronecker sequence for almost all $\alpha$ we have

$$
N D_{N}^{*}=\mathcal{O}\left((\log N)^{1+\varepsilon}\right) \text { for all } \varepsilon>0,
$$

which is close to optimality since by a classical result of W. M. Sc h m id the discrepancy of every infinite sequence satisfies

$$
N D_{N}^{*}=\Omega(\log N) .
$$

For sequences of the form $\left(\left\{a_{n} \alpha\right\}\right)_{n \geq 1}$, R. C. B a k er [4] has shown the following general metric result. Let $\left(a_{n}\right)_{n \geq 0}$ be a strictly increasing sequence of integers. Then for almost all $\alpha$ for the discrepancy of $\left(\left\{a_{n} \alpha\right\}\right)_{n \geq 1}$ we have

$$
N D_{N}^{*}=\mathcal{O}\left(N^{\frac{1}{2}}(\log N)^{\frac{3}{2}+\varepsilon}\right) \text { for all } \varepsilon>0 .
$$

This result is best possible, up to logarithmic factors, since, for example, for lacunary sequences $\left(a_{n}\right)_{n \geq 1}$, that is for sequences satisfying $\frac{a_{n+1}}{a_{n}} \geq 1+\delta$ with $\delta>0$, we have

$$
N D_{N}^{*}=\Omega\left(N^{\frac{1}{2}}\right)
$$

Indeed in this case much sharper results are known - see, for example, 9] or [13]. For general sequences $\left(a_{n}\right)_{n>1}$ which grow neither linearly nor exponentially it is usually very hard to find the correct metric order of the discrepancy of $\left(\left\{a_{n} \alpha\right\}\right)_{n \geq 1}$, and in particular only very few metric lower bounds are known. There are some notable exceptions, such as, for example, [5] and [6], but they usually are either very restrictive or depend on a strong arithmetic structure and deep number-theoretic tools. In [2] the authors of the present paper developed a new, fairly general method by which one can obtain metric lower bounds for the discrepancy of sequences of the form $\left(\left\{a_{n} \alpha\right\}\right)_{n>1}$. Amongst other results, for example, the following was shown there (Corollary 1 in [2]). Let $P \in \mathbb{Z}[x]$ be a polynomial of degree $d \geq 2$. Then for the discrepancy of the sequence $(\{P(n) \alpha\})_{n \geq 1}$ we have for almost all $\alpha$

$$
N D_{N}^{*}=\Omega\left(N^{\frac{1}{2}-\varepsilon}\right) \quad \text { for all } \varepsilon>0 .
$$

Together with the general upper bounds of B aker this means that for these sequences we have the essentially largest possible metric order of $D_{N}^{*}$, namely $N D_{N}^{*} \approx N^{\frac{1}{2}}$. At this point one might assume that for any choice of $\left(a_{n}\right)_{n \geq 1}$ the discrepancy of $\left(\left\{a_{n} \alpha\right\}\right)_{n \geq 1}$ for almost all $\alpha$ either satisfies $N D_{N}^{*}=\mathcal{O}\left(N^{\varepsilon}\right)$ or $N D_{N}^{*}=\Omega\left(N^{\frac{1}{2}-\varepsilon}\right)$. This, however, is not true: in [3] it was shown that any asymptotic order for $N D_{N}^{*}$ between $N^{\varepsilon}$ and $N^{\frac{1}{2}}$ is possible for almost all $\alpha$. More precisely, Theorem 1 in [3] states the following. Let $0<\gamma \leq \frac{1}{2}$ be given. 


\section{ADDITIVE ENERGY AND IRREGULARITIES OF DISTRIBUTION}

Then there exists a strictly increasing sequence $\left(a_{n}\right)_{n \geq 1}$ of positive integers such that for the discrepancy of $\left(\left\{a_{n} \alpha\right\}\right)_{n \geq 1}$ for almost all $\alpha$ we have $N D_{N}^{*}=\mathcal{O}\left(N^{\gamma}\right)$ and $N D_{N}^{*}=\Omega\left(N^{\gamma-\varepsilon}\right)$ for all $\varepsilon>0$. An even more precise result has been recently obtained by $\mathrm{F} \mathrm{u} \mathrm{k} \mathrm{u} \mathrm{y} \mathrm{a} \mathrm{m}$ a and $\mathrm{N}$ is hi m u r a [10], by using a randomization technique.

The purpose of the present paper is to point out that the so-called additive energy of the sequence $\left(a_{n}\right)_{n \geq 1}$ of integers can give some information on the metric distribution behaviour of $\left(\left\{a_{n} \alpha\right\}\right)_{n>1}$. More precisely, we will show that an upper bound for the additive energy of $\left(a_{n}\right)_{n \geq 1}$ implies a lower bound for the metric discrepancy of $\left(\left\{a_{n} \alpha\right\}\right)_{n>1}$, under an additional, relatively moderate, growth assumption on $\left(a_{n}\right)_{n \geq 1}$. The additive energy $E(A)$ of finite sets $A$ of integers was studied very intensively in recent years, especially in connection with additive combinatorics (see, for example, [15]). For many classes of sequences, good upper bounds for the additive energy are known. Thus the link between additive energy and metric discrepancy allows us to identify many interesting classes of integer sequences $\left(a_{n}\right)_{n \geq 1}$ for which we can give good lower bounds for the metric discrepancy of $\left(\left\{a_{n} \alpha\right\}\right)_{n \geq 1}$.

Let $A=\left\{b_{1}, \ldots, b_{N}\right\}$ a set of integers. Then the additive energy $E(A)$ is defined by

$$
E(A):=\#\left\{\left(x_{1}, x_{2}, x_{3}, x_{4}\right) \in A^{4} \mid x_{1}-x_{2}=x_{3}-x_{4}\right\},
$$

i.e., $E(A)$ is the number of solutions of the equation $x_{1}-x_{2}=x_{3}-x_{4}$ with $x_{1}, x_{2}, x_{3}, x_{4} \in A$. If the elements $b_{1}, \ldots, b_{N}$ of $A$ are pairwise distinct, then obviously we always have $N^{2} \leq E(A) \leq N^{3}$. In the present paper we will prove the following theorem, which allows us to deduce lower bounds for the metric discrepancy from upper bounds for the additive energy.

TheOREM 1. Let $\left(a_{n}\right)_{n \geq 1}$ be a strictly increasing sequence of positive integers with $a_{n} \ll e^{\gamma(\log n)^{2}}$ for some $\gamma>0$. Assume furthermore that

$$
E\left(\left\{a_{1}, \ldots, a_{N}\right\}\right) \ll N^{\kappa} \quad \text { for some } \kappa \in[2,3] .
$$

Then for the discrepancy of the sequence $\left(\left\{a_{n} \alpha\right\}\right)_{n \geq 1}$ for almost all $\alpha$ we have

$$
N D_{N}^{*}=\Omega\left(N^{\frac{3-\kappa}{2}-\varepsilon}\right) \quad \text { for all } \varepsilon>0 .
$$

Our result shows in particular that for $\left(a_{n}\right)_{n \geq 1}$ with smallest possible additive energy, i.e., in the case $\kappa=2$, the sequence $\left.\left({ }_{n} \alpha\right\}\right)_{n \geq 1}$ for almost all $\alpha$ has essentially the largest possible discrepancy. More precisely, the following corollary holds. 


\section{CHRISTOPH AISTLEITNER — GERHARD LARCHER}

CoRollaRY 1. Let $\left(a_{n}\right)_{n \geq 1}$ be a strictly increasing sequence of positive integers for which $a_{n} \ll e^{\gamma(\log N)^{2}}$ for some $\gamma>0$ and

$$
E\left(\left\{a_{1}, \ldots, a_{N}\right\}\right)=\mathcal{O}\left(N^{2+\varepsilon}\right) \quad \text { for all } \quad \varepsilon>0 .
$$

Then for the discrepancy of $\left(\left\{a_{n} \alpha\right\}\right)_{n \geq 1}$ for almost all $\alpha$ we have $N D_{N}^{*}=$ $\mathcal{O}\left(N^{\frac{1}{2}+\varepsilon}\right)$ and $N D_{N}^{*}=\Omega\left(N^{\frac{1}{2}-\varepsilon}\right)$ for all $\varepsilon>0$.

Our proof of Theorem 1 is essentially just a slight extension of the proof of Theorem 3 in 2. However, as already noted, the fact that Theorem 1 in the present paper is formulated in the language of additive combinatorics allows us to use several pre-existing results on the additive energy of integer sequences to obtain lower metric bounds for the discrepancy of $\left(\left\{a_{n} \alpha\right\}\right)_{n \geq 1}$ for several interesting specific examples of sequences $\left.a_{n}\right)_{n \geq 1}$. So, for example, we can deduce the following results.

TheOREM 2. Let $\left(a_{n}\right)_{n \geq 1}$ be a sequence of integers with $a_{n} \ll e^{\gamma(\log n)^{2}}$ for some $\gamma>0$ which is convex, i.e., which satisfies

$$
a_{n+1}-a_{n}>a_{n}-a_{n-1} \quad \text { for } \quad n \geq 2 .
$$

Then for almost all $\alpha$ for the discrepancy of the sequence $\left(\left\{a_{n} \alpha\right\}\right)_{n \geq 1}$ we have

$$
N D_{N}^{*}=\Omega\left(N^{\frac{7}{26}-\varepsilon}\right) \text { for all } \varepsilon>0 .
$$

Proof of Theorem 2, The result follows immediately from Theorem 1 above and from Theorem 1 in [14, which states that for every convex set $A$ of $N$ elements we have

$$
E(A) \ll N^{\frac{32}{13}}(\log N)^{\frac{71}{65}} .
$$

Theorem 3. Let $a_{n}=\lfloor F(n)\rfloor$, where the real-valued function $F$ is three times continuously differentiable on $[1, \infty)$ and satisfies

$$
F^{\prime}(x)>0, \quad F^{\prime \prime}(x)>0, \quad \text { and } \quad F^{\prime \prime \prime}(x)<0
$$

on $[1, \infty)$. Then for almost all $\alpha$ for the discrepancy of the sequence $\left(\left\{a_{n} \alpha\right\}\right)_{n \geq 1}$ we have

$$
N D_{N}^{*}=\Omega\left(N^{\min \left(\frac{1}{4}, \frac{1+\rho_{F}}{2}\right)-\varepsilon}\right) \text { for all } \varepsilon>0 \text {, where } \rho_{F}:=\liminf _{N \rightarrow \infty} \frac{\log F^{\prime \prime}(N)}{\log N} .
$$

Proof of Theorem 3. The result follows immediately from Theorem 1 above and from Corollary 2 in [1] which shows that

$$
E\left(\left\{a_{1}, \ldots, a_{N}\right\}\right) \ll N^{\frac{5}{2}}+\frac{N^{2} \log N}{F^{\prime \prime}(N)} \text { for all } N .
$$




\section{ADDITIVE ENERGY AND IRREGULARITIES OF DISTRIBUTION}

From Theorems 2 and 3 we immediately obtain the following examples:

Corollary 2. Let $a_{n}:=\left\lfloor n^{c}\right\rfloor$ for some $c \in(1, \infty)$. Then for almost all $\alpha$ for the discrepancy of the sequence $\left(\left\{a_{n} \alpha\right\}\right)_{n \geq 1}$ we have

$$
N D_{N}^{*}=\Omega\left(N^{\tau-\varepsilon}\right) \quad \text { for all } \varepsilon>0
$$

where

$$
\tau=\left\{\begin{array}{cll}
\frac{c-1}{2} & \text { if } \quad 1<c<\frac{3}{2}, \\
\frac{1}{4} & \text { if } \quad \frac{3}{2} \leq c<2, \\
\frac{7}{26} & \text { if } \quad c \geq 2 .
\end{array}\right.
$$

CoRollaRy 3. Let $a_{n}:=\left\lfloor e^{\gamma(\log N)^{\beta}} \mid\right.$ for some $\gamma>0$ and $\beta$ with $1<\beta \leq 2$. Then for almost all $\alpha$ for the discrepancy of $\left(\left\{a_{n} \alpha\right\}\right)_{n \geq 1}$ we have

$$
N D_{N}^{*}=\Omega\left(N^{\frac{7}{26}-\varepsilon}\right) \quad \text { for all } \varepsilon>0 .
$$

As already mentioned above, for the additive energy $E(A)$ of a finite set $A$ of distinct integers we always have $|A|^{2} \leq E(A) \leq|A|^{3}$, and for every strictly increasing sequence $\left(a_{n}\right)_{n \geq 1}$ of positive integers for almost all $\alpha$ the order of the discrepancy of $\left(\left\{a_{n} \alpha\right\}\right)_{n \geq 1}$ essentially is between $N^{\varepsilon}$ and $N^{\frac{1}{2}}$. The quintessence of Theorem 1 is that a small order of the additive energy of $\left\{a_{1}, \ldots, a_{N}\right\}$ for all $N$ implies a large metric order of $N D_{N}^{*}$ for $\left(\left\{a_{n} \alpha\right\}\right)_{n>1}$. In particular, the lowest possible order of the additive energy of $\left\{a_{1}, \ldots, a_{N}\right\}$ for all $N$ implies the largest possible metric order of $N D_{N}^{*}$ of $\left(\left\{a_{n} \alpha\right\}\right)_{n \geq 1}$.

It is tempting to ask whether the converse statement also is true, that is whether a large order of the additive energy of $\left\{a_{1}, \ldots, a_{N}\right\}$ for all $N$ necessarily implies a small metric order of $N D_{N}^{*}$ of $\left(\left\{a_{n} \alpha\right\}\right)_{n>1}$. This hypothesis seems to be supported by the pure Kronecker sequence $(\{n \alpha\})_{n>1}$, i.e., $a_{n}=n$. The additive energy in this case satisfies $E\left(\left\{a_{1}, \ldots, a_{N}\right\}\right) \gg N^{3}$, so it is of the largest possible order, and $N D_{N}^{*}=\mathcal{O}\left(N^{\varepsilon}\right)$ for all $\varepsilon>0$ for almost all $\alpha$, which means that the discrepancy is of the lowest possible order.

However, the hypothesis is not true, as the following example given in Theorem 4 shows. There we present a sequence $\left(a_{n}\right)_{n \geq 1}$ which has both the largest possible order of the additive energy as well as the largest possible metric order of the discrepancy for $\left(\left\{a_{n} \alpha\right\}\right)_{n \geq 1}$. This sequence $\left(a_{n}\right)_{n \geq 1}$ is characterized by the Rudin-Shapiro sequence. 


\section{CHRISTOPH AISTLEITNER — GERHARD LARCHER}

Rudin-Shapiro sequence $\left(r_{0}, r_{1}, r_{2}, \ldots\right)=(1,1,1,-1,1,1,-1,1, \ldots)$ is defined by $r_{k}=\left\{\begin{aligned} 1 & \text { if the number of } 11 \text {-blocks in the base } 2 \text { representation of } k \text { is even, } \\ -1 & \text { otherwise. }\end{aligned}\right.$

Let the sequence $\left(a_{n}\right)_{n \geq 1}=(0,1,2,4,5,7, \ldots)$ be the sequence of those indices $k$ for which $r_{k}=1$ in the Rudin-Shapiro sequence, sorted in increasing order. We will call this sequence the sequence of Rudin-Shapiro integers. By construction this sequence is strictly increasing.

TheOREM 4. Let $\left(a_{n}\right)_{n \geq 1}$ be the sequence of Rudin-Shapiro integers. Then the additive energy of $\left\{a_{1}, \ldots, a_{N}\right\}$ is of maximal possible order, i.e.,

$$
E\left(\left\{a_{1}, \ldots, a_{N}\right\}\right) \gg N^{3},
$$

and for almost all $\alpha$ the discrepancy of $\left(\left\{a_{n} \alpha\right\}\right)_{n \geq 1}$ is also essentially of maximal possible order, i.e.,

$$
N D_{N}^{*}=\Omega\left(N^{\frac{1}{2}-\varepsilon}\right) \quad \text { for all } \varepsilon>0 .
$$

This result should be compared to another example which was given in [1]. There the sequence $\left(a_{n}\right)_{n \geq 1}$ of Thue-Morse integers (also called evil numbers) was studied. This sequence also has additive energy of maximal possible order, and in this case for almost all $\alpha$ for the discrepancy of $\left(\left\{a_{n} \alpha\right\}\right)_{n \geq 1}$ we have $N D_{N}^{*}=\mathcal{O}\left(N^{0.4035}\right)$ and $N D_{N}^{*}=\Omega\left(N^{0.4033}\right)$.

It remains to prove Theorems 1 and Theorem 4 . These proofs will be given in the next section.

\section{The proofs of Theorem 1 and of Theorem 4}

Proof of The or em 11. For a strictly increasing sequence $\left(a_{n}\right)_{n \geq 1}$ of positive integers we set $I(N):=\int_{0}^{1}\left|\sum_{n=1}^{N} e^{2 \pi i a_{n} \alpha}\right| d \alpha$, and we write $E\left(A_{N}\right)$ for the additive energy of $A_{N}:=\left\{a_{1}, \ldots, a_{N}\right\}$. Note that by orthogonality we have

$$
E\left(A_{N}\right)=\int_{0}^{1}\left|\sum_{n=1}^{N} e^{2 \pi i a_{n} \alpha}\right|^{4} d \alpha
$$

By a classical trick, which is based on an application of Hölder's inequality, we have $I(N) \geq\left(\frac{N^{3}}{E\left(A_{N}\right)}\right)^{\frac{1}{2}}$ (see, for example, [12, Theorem 1]). Hence, if $E\left(A_{N}\right) \ll N^{\kappa}$, then

$$
I(N) \gg N^{\frac{3-\kappa}{2}}
$$




\section{ADDITIVE ENERGY AND IRREGULARITIES OF DISTRIBUTION}

In [2, Theorem 3] the following result $(*)$ was shown:

Let $\left(a_{n}\right)_{n \geq 1}$ be a sequence of integers such that for some $t \in \mathbb{N}$ we have $\left|a_{n}\right| \leq n^{t}$ for all $n$ large enough. Assume there exist a number $\tau \in(0,1)$ and a strictly increasing sequence $\left(B_{L}\right)_{L \geq 1}$ of positive integers with $\left(B^{\prime}\right)^{L} \leq B_{L} \leq B^{L}$ for some reals $B^{\prime}, B$ with $1<B^{\prime}<B$, such that for all $\varepsilon>0$ and all $L>L(\varepsilon)$ we have $I\left(B_{L}\right)>B_{L}^{\tau-\varepsilon}$. Then for almost all $\alpha \in[0,1)$ for all $\varepsilon>0$ for the discrepancy $D_{N}^{*}$ of the sequence $\left(\left\{a_{n} \alpha\right\}\right)_{n \geq 1}$ we have $N D_{N}^{*}=\Omega\left(N^{\tau-\varepsilon}\right)$.

The proof of this result was based on a further result $(* *)$, which is stated as Theorem 4 in [2]:

Let $\left(R_{L}\right)_{L \geq 0}$ be a sequence of measurable subsets of $[0,1)$, with the measure $\mathbb{P}\left(R_{L}\right)$ of $R_{L}$ satisfying $\mathbb{P}\left(R_{L}\right) \geq \frac{1}{B^{L}}$ for some constant $B>0$, and such that each $R_{L}$ is the disjoint union of at most $A^{L}$ intervals for some $A>0$. Then for almost all $\alpha \in[0,1)$ for every $\eta>0$ there are infinitely many integers $h_{L}$ with $h_{L} \leq(1+\eta)^{L} \frac{1}{\mathbb{P}\left(R_{L}\right)}$ and $\left\{h_{L} \alpha\right\} \in R_{L}$.

It turns out that this last result $\left({ }^{*}\right)$ is also true in a stronger version, namely under the weaker assumption that $R_{L}$ is the disjoint union of at most $A^{L^{2}}$ intervals for some $A>0$. This can easily be seen by the following proof of Theorem 4 in [2] line by line, replacing $A^{L}$ by $A^{L^{2}}$ and choosing the value $G_{L}$ which appears in the proof as $G_{L}:=A^{2 L^{2}}(B(1+\eta))^{2 L}$ instead of $G_{L}=(A B(1+\eta))^{2 L}$.

From this a stronger version of $(*)$ follows, namely the fact that the conclusion of $\left(^{*}\right)$ also holds under the weaker assumption that $a_{n}<e^{\gamma(\log n)^{2}}$ for some $\gamma>0$. This can also be easily seen by the following proof of Theorem 3 in [2] line by line. We just have to change formula (18) in this proof in [2] to

$$
\begin{aligned}
\left|f_{L}\left(\alpha_{1}\right)-f_{L}\left(\alpha_{2}\right)\right| & \leq 2 \pi B_{L} e^{\gamma\left(\log B_{L}\right)^{2}} \\
& \leq 2 \pi B^{L} e^{\gamma L^{2}(\log B)^{2}} \\
& \ll A^{L^{2}}
\end{aligned}
$$

for some constant $A>1$. As a consequence the function $g_{L}$ appearing in the proof can be written as a sum of $\ll A^{L^{2}}$ indicator functions of disjoint intervals, and hence the set $M_{L}^{\left(i_{L}\right)}$ appearing in the proof is always a union of $\ll A^{L^{2}}$ intervals. Then the stronger version of $(* *)$ which we have obtained above is used to establish the stronger version of $(*)$. Then the desired result follows immediately from this stronger version of $\left(^{*}\right)$ together with (1). 


\section{CHRISTOPH AISTLEITNER — GERHARD LARCHER}

Proof of Theorem 目 Let $\rho_{n}(x):=\sum_{k=0}^{2^{n}-1} r_{k} x^{k}$ be the Rudin-Shapiro polynomials. From Theorem 2.1 in [8] it follows that

$$
\int_{0}^{1}\left|\rho_{n}\left(e^{2 \pi i \alpha}\right)\right| d \alpha \gg 2^{\frac{n}{2}} \text { for all } n .
$$

Let

then

$$
\sum(n):=\sum_{k=0}^{2^{n}-1} e^{2 \pi i a_{k} \alpha}
$$

$$
\sum(n)=\frac{1}{2}\left(\rho_{n}\left(e^{2 \pi i \alpha}\right)+\sum_{k=0}^{2^{n}-1} e^{2 \pi i k \alpha}\right)
$$

We have

$$
\begin{aligned}
\int_{0}^{1}\left|\sum_{k=0}^{2^{n}-1} e^{2 \pi i k \alpha}\right| d \alpha & \leq \int_{0}^{1} \min \left(2^{n}, \frac{1}{\|\alpha\|}\right) d \alpha \\
& \leq 2+2 \int_{\frac{1}{2^{n}}}^{\frac{1}{2}} \frac{1}{\alpha} d \alpha \\
& \leq 2+2 n .
\end{aligned}
$$

Hence

$$
\int_{0}^{1}\left|\sum_{k=0}^{2^{n}-1} e^{2 \pi i a_{k} \alpha}\right| d \alpha \gg \int_{0}^{1}\left|\rho_{n}\left(e^{2 \pi i \alpha}\right)\right| d \alpha-\int_{0}^{1}\left|\sum_{k=0}^{2^{n}-1} e^{2 \pi i k \alpha}\right| d \alpha \gg 2^{\frac{n}{2}} .
$$

It is also well known (see, for example, [7]) that we always have

and therefore

$$
\sqrt{\frac{3 l}{5}}<\sum_{k=0}^{l-1} r_{k}<\sqrt{6 l}
$$

$$
a_{k} \leq 2 k \text { for all } k \text {. }
$$

From (44) we immediately obtain $E\left(\left\{a_{1}, \ldots, a_{N}\right\}\right) \gg N^{3}$, and from (3) and (4) and using Theorem [3 in [2] we obtain the desired $\Omega$-estimate for $N D_{N}^{*}$.

\section{REFERENCES}

[1] AistleitneR, C.-HOFER, R.-LARCHER, G.: On Evil Kronecker Sequences and Lacunary Trigonometric Products, To appear in: Annales del institute Fourier. 2016. http://arxiv.org/abs/1502.06738.

[2] AISTLEITNER, C.-LARCHER, G.: Metric results on the discrepancy of sequences $\left(a_{n} \alpha\right)_{n \geq 1}$ modulo one for integer sequences $\left(a_{n}\right)_{n \geq 1}$ of polynomial growth, Mathematika 62 (2016), 478-491. 


\section{ADDITIVE ENERGY AND IRREGULARITIES OF DISTRIBUTION}

[3] AISTLEITNER, C.-LARCHER, G.: On sequences with prescribed metric discrepancy behavior, Monatsh. Math. 181 (2016), 507-514.

[4] BAKER, R. C.: Metric number theory and the large sieve, J. London Math. Soc. (2) 24 (1981), no.1, 34-40.

[5] BERKES, I.-PHILIPP, W.: The size of trigonometric and Walsh series and uniform distribution mod 1, J. London Math. Soc. (2) 50 (1994), no. 3, 454-464.

[6] BERKES, I.-PHILIPP, W.-TICHY, R. F.: Empirical processes in probabilistic number theory: the LIL for the discrepancy of $\left(n_{k} \omega\right) \bmod 1$, Illinois J. Math. 50 (2006), no. 1-4, 107-145.

[7] BRILlharT, J.-MORTON, P.: A Case Study in Mathematical Research: The GolayRudin-Shapiro Sequence, Amer. Math. Monthly 103 (1996), 854-869.

[8] ERDÉLYI, T.: The Mahler Measure of the Rudin-Shapiro Polynomials, Constr. Approx. (to appear); http://arxiv.org/abs/1406.2233

[9] FUKUYAMA, K.: The law of the iterated logarithm for discrepancies of $\left\{\theta^{n} x\right\}$, Acta Math. Hungar. 118 (2008), no. 1-2, 155-170.

[10] FUKUYAMA, K.-NISHIMURA, T.: A metric discrepancy result with given speed. Preprint.

[11] GARAEV, M. Z.: Upper bounds for the number of solutions of a diophantine equation. Transactions Amer. Math. Soc. 357 (2004), 2527-2534.

[12] KARATSUBA, A. A.: An estimate of the $L_{1}$-norm of an exponential sum. Math. Notes 64 (1998), 401-404.

[13] PHILIPP, W.: Limit theorems for lacunary series and uniform distribution mod 1. Acta Arith. 26 (1974/75), no. 3, 241-251.

[14] SHKREDOV, I. D.: Some new results on higher energies. Trans. Moscow Math. Soc. 74 (2013), 31-63.

[15] TAO, T.-VU. V.: Additive Combinatorics. Cambridge University Press, Cambridge U.K., 2006.

Received March 7, 2016

Accepted March 17, 2016

\section{Christoph Aistleitner \\ Gerhard Larcher}

Institute of Financial Mathematics and Applied Number Theory

University Linz

Altenbergerstraße 69

A-4040-Linz

AUSTRIA

E-mail: christoph.aistleitner@jku.at gerhard.larcher@jku.at 